\title{
The Role of the Magnesium Valproate in Therapy for Patients with Treatment-Resistant Depression (TRD): Meat-Analysis Results of Chinese Data
}

\author{
Chen Fengpei ${ }^{1}$, Zhu Jianfeng', Gao Zhihan ${ }^{1}$, Shen Yin ${ }^{2}$, Jin Rui ${ }^{3}$, Ren Zhibin ${ }^{2}$, Chen Zhenxin ${ }^{2}$, \\ Ma Yongchun ${ }^{2}$, Jin Weidong 1,2 , * \\ ${ }^{1}$ The Graduate School, Zhejiang Chinese Medicine University, Hangzhou, China \\ ${ }^{2}$ Department of Psychiatry, Tongde Hospital Affiliated to Zhejiang Chinese Medicine University, Zhejiang Province Tongde Hospital, Zhejiang \\ Province Mental Health Institute, Hangzhou, China \\ ${ }^{3}$ Department of Pharmacology, Tongde Hospital Affiliated to Zhejiang Chinese Medicine University, Zhejiang Province Tongde Hospital, \\ Hangzhou, China
}

\section{Email address: \\ wdjin@163.com (Jin Weidong), 839158980@qq.com (Zhu Jianfeng), 574892686@qq.com (Gao Zhihan), 1023937809@qq.com (Chen Fengpei), Shenwind@hotmail.com (Shen Yin),jwdjinrui@126.com (Jin Rui), renzhib1996@sina.com (Ren Zhibin),toteczx@msn (Chen Zhenxin), Yongchun3@sina.com (Ma Yongchun) \\ ${ }^{*}$ Corresponding author}

\section{To cite this article:}

Chen Fengpei, Zhu Jianfeng, Gao Zhihan, Shen Yin, Jin Rui, Ren Zhibin, Chen Zhenxin, Ma Yongchun, Jin Weidong. The Role of the Magnesium Valproate in Therapy for Patients with Treatment-Resistant Depression(TRD): Meat-Analysis Results of Chinese Data. International Journal of Pharmacy and Chemistry. Vol. 4, No. 3, 2018, pp. 23-30. doi: 10.11648/j.jppc.20180403.11

Received: October 10, 2018; Accepted: November 5, 2018; Published: December 3, 2018

\begin{abstract}
Background: Major depressive disorder is a prevalent and disabling illnessv, approximately $70 \%$ of patients do not remit after first-line antidepressant treatment, and about $20 \%$ develop to treatment-resistant depression(TRD). Treatment of TRD have been one of clinical problems. Adjunctive valprovate was a most sued ways for treatment of TRD. The magnesium valproate was common drug for both episode and bipolar disorder in China and also was used for TRD. To systematically evaluate the difference in efficacy of magnesium valproate and antidepressants for therapy of treatment-resistant depression(TRD) in China. Methods: Searches were applied to the following electronic databases in china: Chinese Biomedical Database (CBM), China National Knowledge Infrastructure (CNKI), WANFANG and Chinese Social Sciences Citation Index (VIP) databases. A total of 13 RCTs were included. A meta-analysis was performed of all the literatures germane to estimate the treatment-resistant depression patients treated with magnesium valproate combination with antidepressants and only antidepressants randomized controlled trials (RCTs) from 2010 to 2015. Odds ratios (ORs) and 95\% confidence intervals (CIs) were calculated and the meta-analysis was conducted with Revman4.2 software. Results: A total of 13 RCTs were included for meta-analysis. The results of meta-analysis demonstrated by effective rate and depressive symptoms change between two groups of combination treatment with magnesium valproate and only antidepressant. The effective rate was higher in magnesium valproate combination with antidepressants group than that in antidepressants group $(81 / 306,35 / 298, Z=4.60$, $\mathrm{P}=0.00001$ ). The depressive scale was lower found in magnesium valproate combination with antidepressants group than that in antidepressants group after treatment $(\mathrm{Z}=16.20, \mathrm{P}<0.00001)$. These results indicated that magnesium valproate combination with antidepressants maybe one better therapeutic way for TRD. Conclusion: The results indicate that magnesium valproate combination with antidepressants was better than antidepressants group in effective rate and improving depressive symptoms during treatment of patients with TRD. So these results may recommend to the patients with TRD though without the differences between groups.
\end{abstract}

Keywords: Magnesium Valproate, Antidepressants, TRD, Meta-analysis, Chinese Data 


\section{Introductions}

Treatment-resistant depression (TRD) is a term used to describe a case of major depressive disorder that does not seem to respond to conventional depression treatment. Clinical psychiatry coined the term is 1974, when electroconvulsive treatment was used extensively to treat serious depressive disorders that seemed to be immune to cognitive-behavioral therapy (CBT) and early antidepressant medications [1]. With the introduction of more varied antidepressants, the term was amended to describe major depressive illness that does not respond to at least two of the newer antidepressant medications. Up to two thirds of patients with major unipolar depression will not respond to the first medication prescribed. Depression may be considered resistant to treatment when at least two trials with antidepressants from different pharmacologic classes (adequate in dose, duration, and compliance) fail to produce a significant clinical improvement [2]. Studies of treatment-resistant depression have used a variety of definitions. A general consensus is emerging that unipolar major depression is considered resistant or refractory when at least two trials with antidepressants from different pharmacologic classes (adequate in dose, duration, and compliance) fail to produce a significant clinical improvement Before determining that a patient is nonresponsive to an initial trial of antidepressants, he or she should be reassessed to confirm the accuracy of the diagnosis, medication adherence, and whether the depression is being exacerbated by coexisting medical, psychiatric, or psychosocial disorders.[3]

Major depressive disorder is a prevalent and disabling illness. Notwithstanding numerous advances in the pharmacological treatment of depression, approximately $70 \%$ of patients do not remit after first-line antidepressant treatment[2]. How about TRD? Epidemiologic data on treatment resistant depression (TRD) in Asia-Pacific countries are limited. We estimated the incidence of TRD in Taiwan using a cohort of 704,265 adults randomly sampled from Taiwan's National Health Insurance Research database for 2005. TRD was defined as a patient having pharmaceutically treated depression (PTD) not adequately responding to 2 antidepressant (AD) regimens. Among 2751 PTD subjects, 576 (20.94\%, 95\% CI: 19.46, 22.49) developed TRD, a proportion similar to that in North American studies. TRD incidence was $0.82(95 \%$ CI: $0.75,0.89)$ cases $/ 1000$ population in 2005 , increased with age, and was higher in females than in males. SSRI's were the most frequently used ADs. Augmentation with antipsychotics was common. The median time from PTD onset (first AD medication) to TRD onset was 416 days but psychiatrists practicing in Taiwan indicated they would switch within $<=3$ months if an AD medication was not effective. We therefore repeated the analysis with a 6 months cap on time from onset of PTD to TRD. In this supplemental, post-hoc, analysis, 68 PTD subjects, $2.47 \%$, (95\% CI: 1.94, 3.10) developed TRD; i.e., 0.10 (95\% CI: $0.08,0.12$ ) incident cases/1000 population[4].
In Japan, in the year from 1 April 2012 through 31 March 2013, we identified 1143 incident PTD cases among 98,552 eligible subjects, i.e. 11.59 cases/1000 patient-years. Of the PTD patients, $51.4 \%$ were women. Within the 1-year observation interval 137 patients failed more than two antidepressive treatment approaches and thus developed TRD. Though co-morbid conditions and age were similar among PTD and TRD patients, medical costs per patient (patient-year) during their treatment intervals were 1.01 million JPY (0.540 million JPY) in the TRD population and 0.643 JPY million JPY (0.645 million JPY) in the PTD population who did not convert into TRD [5]. In went nations, of the treated episodes, $6.6 \%(\mathrm{~N}=3,134)$ met the criteria for TRD. The median time to an episode becoming TRD was approximately one year. The mean duration of a TRD episode was 1,004 days (vs. 452 days for a non-TRD episode). More than $75 \%$ of TRD episodes had at least four lines of therapy; half of the treatment regimens included a combination of drugs. Average hospitalization costs were higher for TRD than non-TRD episodes [6]

Treatment of TRD have been one of clinical problems. Adjunctive antipsychotics was a most sued ways[4], especially adjunctive aripiprazole showed benefits in improving the response rate, remission rate, and the quality of life in patients with TRD[7]. The success of switching to a different antidepressant following a first-line agent is supported by evidence, but there is limited evidence for effective combination strategies. Lithium and T3 augmentation of TCA have the strongest evidence base for successful treatment of TRD. The use of augmentation of newer-generation antidepressants with atypical antipsychotics is supported by a growing evidence base. Current evidence supports CT as an effective strategy for TRD. There is a need for additional large-scale RCT of TRD. The development of new antidepressants targeting novel pathways opens a promising perspective for the management of TRD [2]. And adjunctive mood stabilizer also common method for TRD[8, 9], especially antidepressant augmentation with VPA provided substantial clinical improvement and maintenance over a relatively long-term period in a subgroup of patients with severe TRD [8].

Magnesium sulfate and sodium valproate both block methylphenidate-induced hyperlocomotion which was an animal model of mania [10]. Especially, magnesium supplementation enhances the anticonvulsant potential of valproate in pentylenetetrazol-treated rats [11]. The magnesium valproate were used as mood stabilizer in China [12]. As adjunctive mood stabilizer also was one of common managements for $\operatorname{TRD}[8,9]$, the Chinese psychiatrists had tried some studies about adjunctive magnesium valproate for treatment TRD. So this analysis assessed these studies by meta-analysis and reported results.

\section{Methods}

This review included randomized controlled trials (both individual and cluster randomisation). We were interested in 
comparisons of adjunctive magnesium valproate treatment of TRD patients. The primary outcome of our meta-analysis were full remission rate, effective rate, changes of depressive symptom,. While the incidence of adverse effect and dropout rate were the second outcome of our meta-analysis.

\subsection{Literature Search and Selection}

Searches were applied to the following electronic databases, but only in china: Chinese Biomedical Database (CBM), China National Knowledge Infrastructure (CNKI), WANFANG and Chinese Social Sciences Citation Index (VIP) databases. The search strategy was based on combinations magnesium valproate treatment and TRD. We also modified the terms according to the different databases. Last query was updated on 31 Des 2017. References of retrieved articles were cross-searched to identify any studies missed by the electronic search strategies.

\subsection{Inclusion and Exclusion Criteria}

Inclusion criteria for original studies were as follows: 1 . The patients involved in trial meet TRD. 2. Trial design was adjunctive magnesium valproate and basic management in treatment for TRD comparing only basic management, regardless of randomized, blind, follow up and publication status. 3. The trial included scale use, full remission rate, effective rate and side events. 4 . The trial observation must last more 4 weeks.5. The paper was published in chinese.

Exclusion criteria for original studies were as follows: 1. when multiple articles were published by the same authors or institutions, the most recent or informative single article was selected. 2. Articles lacking original data for meta-analysis. 3. Review articles .4. Case report.

\subsection{Data Extraction}

The initial selection of all candidate articles was relied on careful screening of their abstracts by two independent reviewers, using a standardized data collection form, including the following items: the first author, year of publication, sex, mean or median age, full remission rate, effective rate, dropout rate, the incidence of adverse effect, clinical symptom score, treatment and control group interventions and assessment of outcomes.

This study manually searched the reference lists of the some articles. We also screened references from the relevant literature, including all of the identified studies, but no additional reviews and editorials. Disagreements were resolved by consensus between the two readers. In case of persistent disagreement, the final decision was made by our expert.

\subsection{Selecting Paper Process}

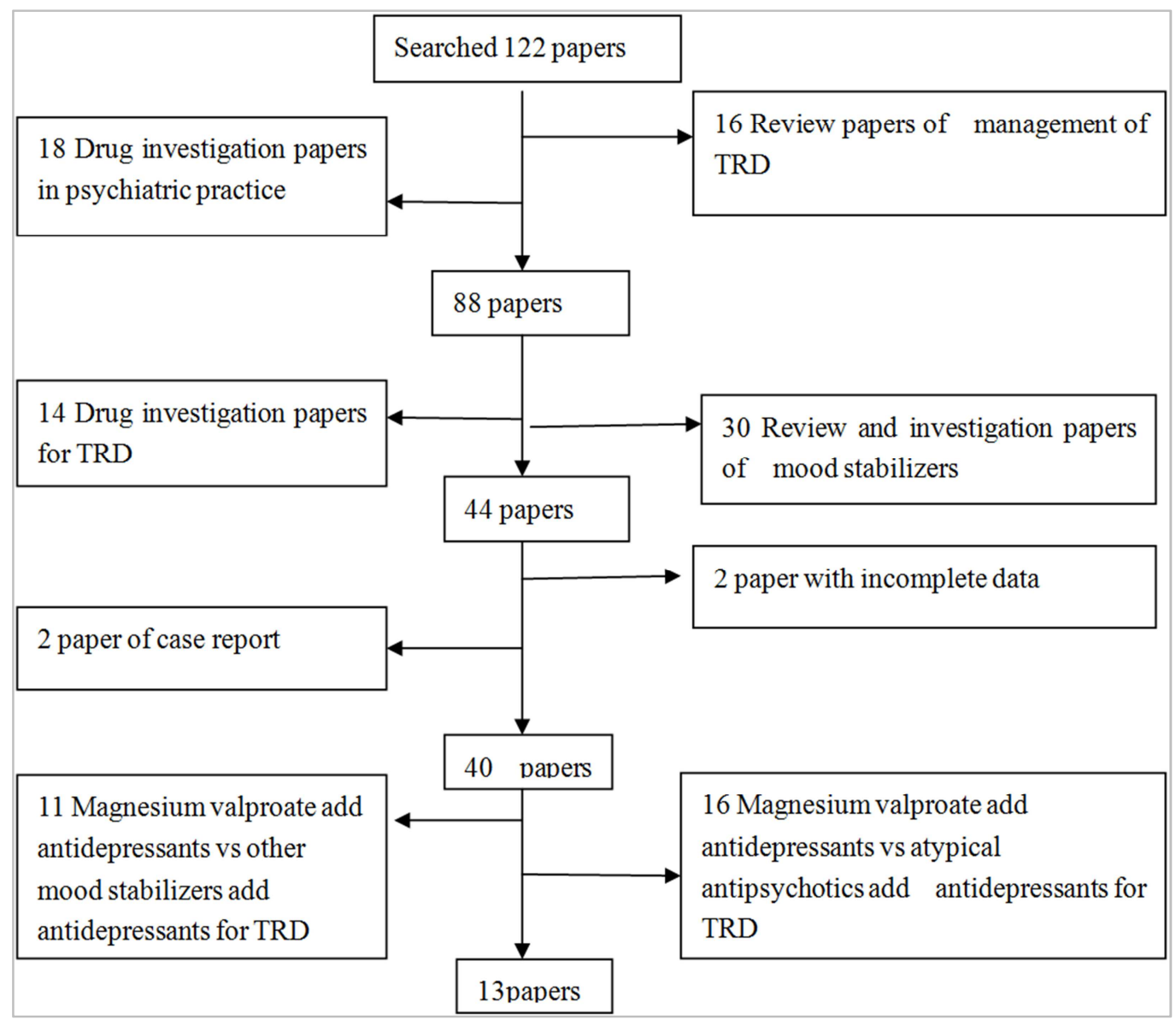

Figure 1. Selecting Paper Precess 
All 122 papers of treatments TRD related to magnesium valproate was found in chiese database. And 16 review paper about treatments of TRD mentioned magnesium valproate and 18 drug investigative paper in psychiatric practice were removed. And among surplus 88 papers, 30 review and investigative paper of mood stabilizers, and 14 drug investigative papers for TRD were also removed. And among surplus 44 papers, 2 papers were incomplete data, and 2 papers were case report. And among surplus 40 papers, 11 paper were designed as magnesium valproate combination with antidepressants comparing to other mood stabilizer combination with antidepressants for treatment of TRD. And 16 paper were designed as magnesium valproate combination with antidepressants comparing to atypical antipsychotics combination with antidepressants for treatment of TRD. The surplus 13 papers meet our criteria that was adjunctive magnesium valproate at basic management in treatment of antidepressant comparing to only basic antidepressant for TRD [13-25].

\subsection{Statistical Methods}

All statistical analyses were performed using Statistical Analysis System software (Revman 4.2), and the P value for the overall effect $<0.05$ with two-tailed was considered statistically significant. The heterogeneity of all involved studies was assessed by $\mathrm{I}^{2}$. When it was lower than $50 \%$, the studies with an acceptable heterogeneity were considered, and then the fixed-effects model with Mantel-Haenszel method was used; otherwise, a random effect model with the Der Simonian and Laird (DL) method was adopted. The combined odds ratio (OR) were initially estimated using Forrest plots raphically. For each trial, the OR was estimated from the original article. If not available, we looked at the total numbers of events and the numbers of patients at risk in each group to determine the OR estimate.

\section{Results}

\subsection{The Effective Rate}

These were 11 articles that report effective rate. 81 cases with effectiveness in magnesium valproate group and 35 cases with effectiveness in control group was found. There was no statistical heterogeneity among the studies $\left(\mathrm{X}^{2}=2.56, \mathrm{df}=10\right.$, $\left.\mathrm{P}=0.99, \mathrm{I}^{2}=0.00 \%\right)$. Thus, the fixed-effect model was used for statistical analysis. The effective rate was higher in magnesium valproate group compared to control group $(81 / 306, \quad 35 / 290, \quad \mathrm{Z}=4.60, \quad \mathrm{P}<0.0001) . \quad$ OR2.04,95CI (1.02 4.43) See Figure 2.

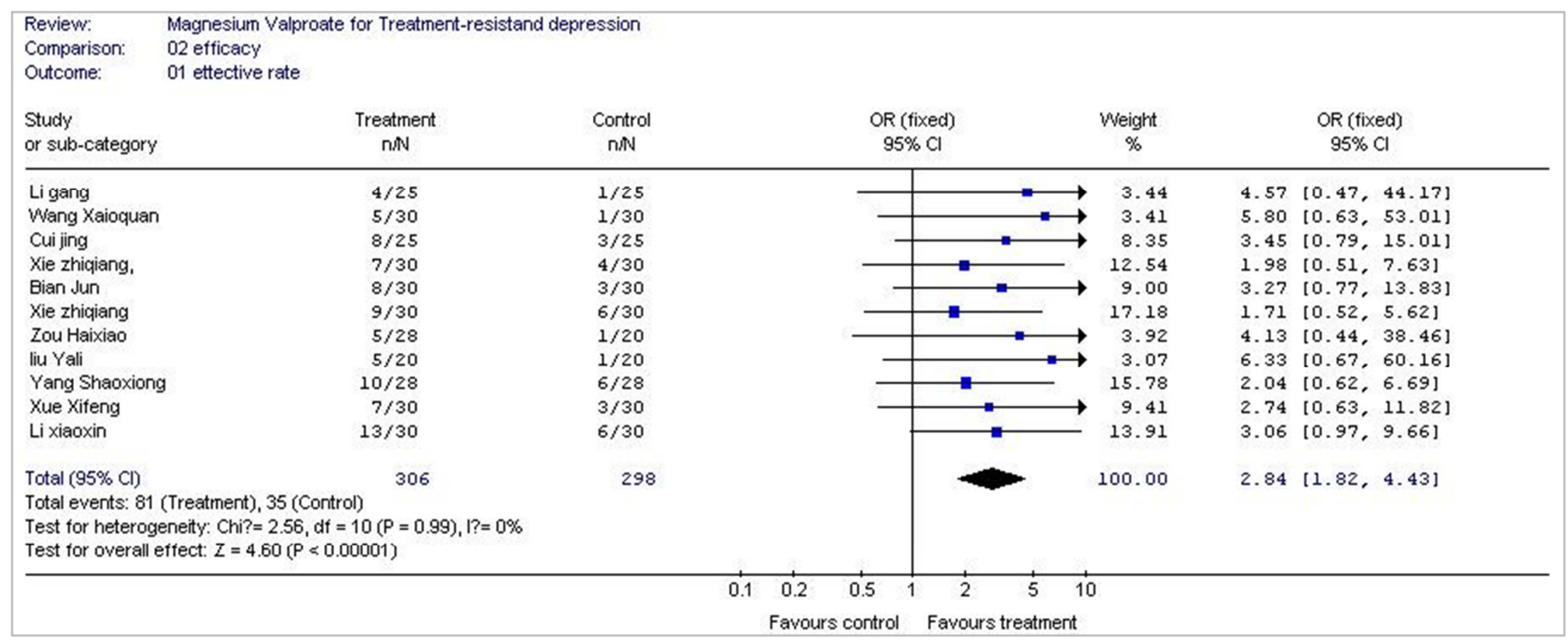

Figure 2. The effective rate between magnesium valproate group and only antidepressants group.

\subsection{Total Changes of Symptoms}

This study observe 4 weeks depressive symptom changes in our analysis. There was statistical heterogeneity among the studies $\left(\mathrm{X}^{2}=123.69, \mathrm{df}=39, \mathrm{P}<0.00001, \mathrm{I}^{2}=68.5 \%\right)$. Thus, the random-effect model was used for statistical analysis. But depressive scale was lower found in magnesium valproate group than that in control group $(Z=16.20, P<0.0001)$. WMD $=-2.64$, 95\% CI (-2.96 - -2.32) See Figure 3. 


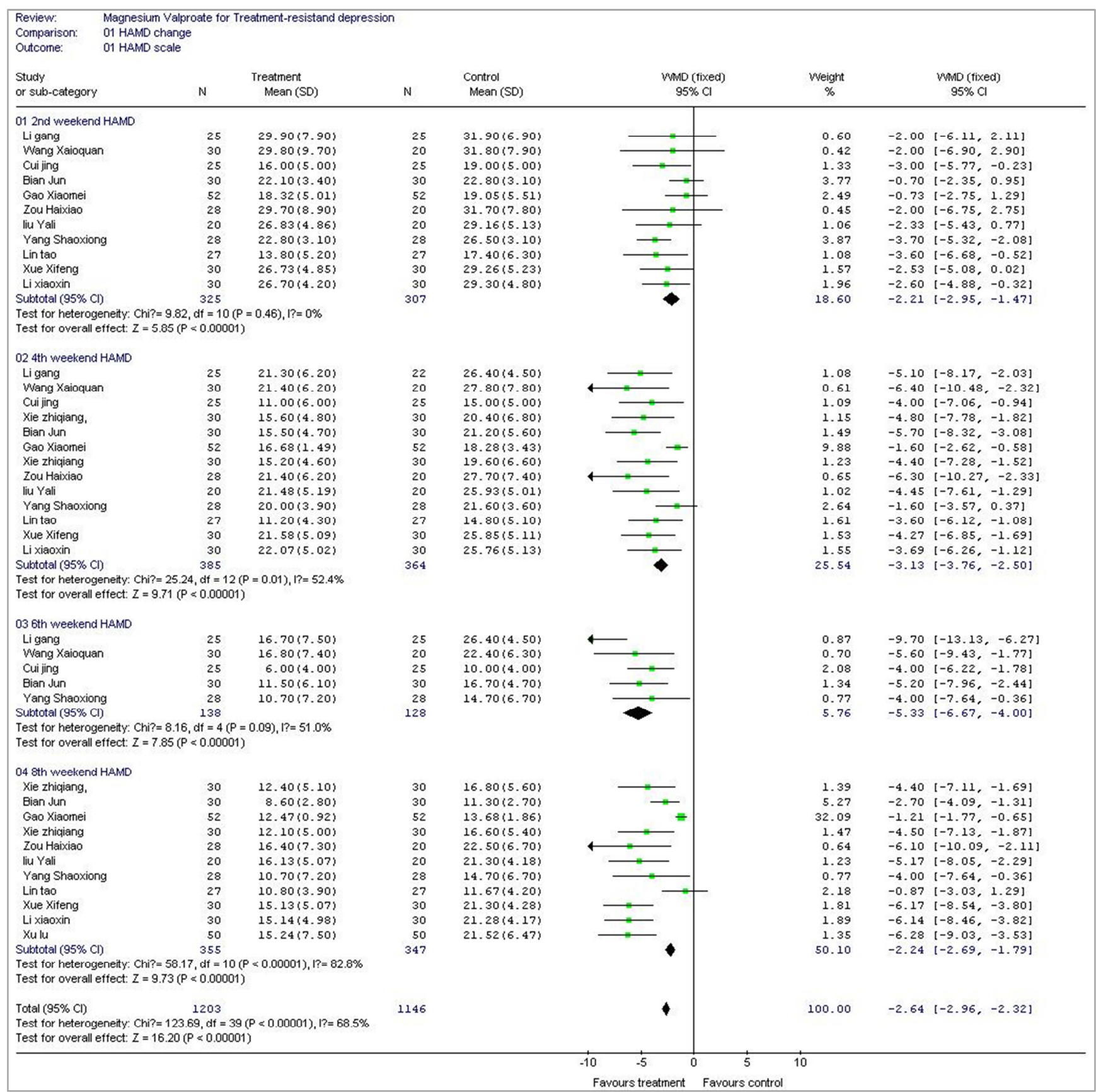

Figure 3. The depressive symptoms changes between magnesium valproate group and only antidepressants group.

\section{Discussion}

The success of switching to a different antidepressant following a first-line agent is supported by evidence, but there is limited evidence for effective combination strategies. Lithium and T3 augmentation of TCA have the strongest evidence base for successful treatment of TRD. The use of augmentation of newer-generation antidepressants with atypical antipsychotics is supported by a growing evidence base. Current evidence supports $\mathrm{CT}$ as an effective strategy for TRD. There is a need for additional large-scale RCT of TRD. The development of new antidepressants targeting novel pathways opens a promising perspective for the management of TRD [2]. And other way for management of TRD is ketamine that is a new antidepressants with pharmaceutical pipeline [26]. Sixty-two trials were identified in US and EU clinical trial registries that included six investigational compounds in recent phase III development and 12 others in recent phase II clinical trials. Glutamatergic agents have been the focus of many studies. A single intravenous dose of the glutamatergic modulator ketamine produces a robust and rapid antidepressant effect in persons with TRD [27]. But ketamine was not been used in clinical practice.

Antidepressant augmentation with VPA may provided substantial clinical improvement and maintenance over a relatively long-term period in a subgroup of patients with 
severe TRD. Ghabrash(2015) observed fourteen TRD patients (seven males and seven females; age 19-59) received VPA (375-1000 mg/day) in addition to their treatment regimen after previously failing to respond to two or more antidepressant trials and/or different combinations. Clinical response to VPA was investigated prior the treatment (T-0) and after 1 (T-1), 4 (T-4) and 7 (T-7) months of therapy[8]. They found compared to T-0, VPA significantly decreased MADRS score at T-1 $(\mathrm{P}<$ $0.001)$, T-4 $(\mathrm{P}<0.001)$ and $\mathrm{T}-7(\mathrm{P}<0.001)($ partial $\eta(2)=0.86)$. Importantly, MADRS score at T-7 $(13.6 \pm 1.6$, mean \pm SEM $)$ was closer to the reported value of remission (MADRS $<10$ ), and none of the patients relapsed during the observational period. Compared to T-0, VPA also decreased CGI-Severity of illness score at T-1 $(\mathrm{p}=0.03), \mathrm{T}-4(\mathrm{p}<0.001)$ and T-7 $(\mathrm{p}<$ $0.001)$ (partial $\eta(2)=0.74)$. And they also suggested that VPA thus deserves further exploration in large double-blind clinical trials. Fang (2015)compared the differences in effectiveness among adjunctive risperidone, valproate, buspirone, trazodone, or thyroid hormone in adult Chinese patients with treatment-resistant major depression[9]. they found the remission rates were $26.7 \%$ for risperidone, $48.7 \%$ for valproate, $32.6 \%$ for buspirone, $42.6 \%$ for trazodone, and $37.5 \%$ for thyroid hormone. There was no statistical significance among treatment arms in remission rates, although remission rate for valproate was highest. These results suggest that VAP may have better effect for TRD. So many studies were carried out in china[13-26]. This also is the reasons that do meta-analysis to found magnesium valproate and antidepressants for therapy of treatment-resistant depression (TRD) in China is better or not. This result found that the effective rate was higher in magnesium valproate group compared to control group (81/306, 35/290, $\mathrm{Z}=4.60$, $\mathrm{P}<0.0001) . \quad \mathrm{OR}=2.04,95 \mathrm{CI}(1.02 \sim 4.43)$ and depressive scale was lower found in magnesium valproate group than that in control group $(\mathrm{Z}=16.20, \quad \mathrm{P} \quad<0.0001)$. $\mathrm{WMD}=-2.64,95 \% \mathrm{CI}(-2.96 \sim-2.32)$. These results show that adjunctive magnesium valproate maybe better in treatment for TRD.

What is the mechanism of mood stabilizers for TRD?. The some study suggests that the acute effect of mood stabilizer lamotrigine augmentation therapy for a major depressive episode is not related to either BDNF or IL-6, at least in patients with treatment-resistant depressive disorder[28]. Chronic administration of the mood stabilizers lithium and divalproex (valproate semisodium) reduces glucocorticoid signaling and function in the hippocampus, which may be one of their mechanisms[29]. Mean 5-HT1A binding potential (BPP) significantly increased following mood stabilizer treatment, most prominently in the mesiotemporal cortex (hippocampus plus amygdala). When mood state was also controlled for, treatment was associated with increases in BPP in widespread cortical areas. These preliminary findings are consistent with the hypothesis that these mood stabilizers enhance 5-HT1A receptor expression in BD, which may underscore an important component of these agents' mechanism of action in TRD[29].

The magnesium valproate maybe have a special action different to sodium valproate. The study have demonstrated that $\mathrm{Mg}(2+)$ could enhance the antiepileptic efficacy of a subprotective dose of VPA, possibly by improving redox balance and modulation of some brain amino acids[30]. Both VPA salts decreased spontaneous neuronal firing activity in greater than $60 \%$ of recorded pyramidal neurons as well as potentiated GABA inhibitions. When injected at equal concentrations and currents, Mg-VPA blocked the excitatory responses induced by N-methyl-D-aspartate (NMDA) more frequently than Na-VPA. Both VPA salts equally blocked the excitatory responses of quisqualate and kainite[31].

This study have some deficiency: (1)limitation of literatures: our data not contained articles in English, and also not contained articles published in Taiwan, Hongkong and Macao. (2)Assessment of articles quality was not done. (3)The side effects of magnesium valproate was estimated.(4)The statistic soft was Revman 4.2 not 5.2. These deficiency maybe influence the level of evidence of magnesium valproate for treatment of TRD.

\section{Acknowledgements}

We thank Mr Zhu Jianfeng do many statistic work for our study and Mr Ma Yongchun made the final amendment of manuscript.

\section{Conflict of Interests}

All authors declare on conflicts of interet with any commencial or other association in connection with submitted article.

Table 1. General information of studied

\begin{tabular}{|c|c|c|c|c|c|c|c|}
\hline \multirow{2}{*}{ Author } & studied & group & control & group & scale & random & blind \\
\hline & Case & drug & case & drug & & & \\
\hline Lin & 27 & MgVAL+citalopram & 27 & citalopram & HAMD HAMA & yes & no \\
\hline $\mathrm{Li}$ & 30 & MgVAL+escitalopram & 30 & escitalopram & HAMD & yes & no \\
\hline Xue & 30 & MgVAL+escitalopram & 30 & escitalopram & HAMD & yes & no \\
\hline Yang & 28 & MgVAL+paroxetine & 28 & paroxetine & HAMD & yes & no \\
\hline Liu & 20 & MgVAL+escitalopram & 20 & escitalopram & HAMD & yes & no \\
\hline Zhou & 28 & MgVAL+escitalopram & 20 & escitalopram & HAMD & yes & no \\
\hline Gao & 52 & MgVAL+duloxetine & 52 & duloxetine & HAMD HAMA & yes & no \\
\hline Bian & 30 & MgVAL+paroxetine & 30 & paroxetine & HAMD & yes & no \\
\hline Cui & 25 & MgVAL+venlafaxine & 30 & venlafaxine & HAMD & yes & no \\
\hline Wang & 30 & MgVAL+sertraline & 20 & sertraline & HAMD & yes & no \\
\hline
\end{tabular}




\begin{tabular}{lllllll}
\hline \multirow{2}{*}{ Author } & studied & group & control & group & scale & random \\
\cline { 2 - 6 } & Case & drug & case & drug & & \\
\hline $\mathrm{Li}$ & 25 & MgVAL+paroxetine & 25 & paroxetine & HAMD & yes \\
$\mathrm{Xie}$ & 30 & MgVAL+venlafaxine & 30 & venlafaxine & HAMD & yes \\
\hline
\end{tabular}

\section{References}

[1] McIntyre RS, Filteau MJ, Martin L, Patry S, Carvalho A, Cha DS, Barakat M, Miguelez M. Treatment-resistant depression: definitions, review of the evidence, and algorithmic approach. J Affect Disord. 2014 Mar; 156: 1-7. doi: 10.1016/j.jad.2013.10.043. Epub 2013 Nov 15.

[2] Carvalho AF, Berk M, Hyphantis TN, McIntyre RS The integrative management of treatment-resistant depression: a comprehensive review and perspectives. Psychother Psychosom. 2014; 83(2): 70-88. doi: 10.1159/000357500. Epub 2014 Jan 22.

[3] Mrazek DA, Hornberger JC, Altar CA, Degtiar I. A review of the clinical, economic, and societal burden of treatment-resistant depression: 1996-2013. Psychiatr Serv. 2014 Aug 1; 65(8): 977-87. doi: 10.1176/appi.ps.201300059.

[4] Fife D, Feng Y, Wang MY, Chang CJ, Liu CY, Juang HT, Furnback W, Singh J, Wang B. Epidemiology of pharmaceutically treated depression and treatment resistant depression in Taiwan. Psychiatry Res. 2017 Jun; 252: 277-283. doi: 10.1016/j.psychres.2017.03.006. Epub 2017 Mar 6.

[5] Mahlich J Tsukazawa S, Wiegand F. Estimating Prevalence and Healthcare Utilization for Treatment-Resistant Depression in Japan: A Retrospective Claims Database Study. Drugs Real World Outcomes. 2017 Nov 28. doi: 10.1007/s40801-017-0126-5. [Epub ahead of print].

[6] Kubitz N1, Mehra M, Potluri RC, Garg N, Cossrow N. Characterization of treatment resistant depression episodes in a cohort of patients from a US commercial claims database. PLoS One. 2013 Oct 18; 8(10): e76882. doi: 10.1371/journal.pone.0076882. eCollection 2013.

[7] Luan S, Wan H, Zhang L, Zhao H. Efficacy, acceptability, and safety of adjunctive aripiprazole in treatment-resistant depression: a meta-analysis of randomized controlled trials. Neuropsychiatr Dis Treat. 2018 Feb 8; 14: 467-477. doi: 10.2147/NDT.S156619. eCollection 2018.

[8] Ghabrash MF, Comai S, Tabaka J, Saint-Laurent M, Booij L, Gobbi G. Valproate augmentation in a subgroup of patients with treatment-resistant unipolar depression. World J Biol

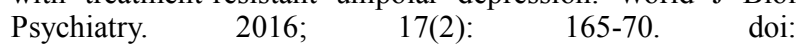
10.3109/15622975.2015.1073856. Epub 2015 Oct 7.

[9] Fang Y, Yuan C, Xu Y, Chen J, Wu Z, Cao L, Yi Z, Hong W, Wang Y, Jiang K, Cui X, Calabrese JR, Gao K. A pilot study of the efficacy and safety of paroxetine augmented with risperidone, valproate, buspirone, trazodone, or thyroid hormone in adult Chinese patients with treatment-resistant major depression. J Clin Psychopharmacol. 2011 Oct; 31(5): 638-42. doi: 10.1097/JCP.0b013e31822bb1d9.

[10] Barbosa FJ, Hesse B, de Almeida RB, Baretta IP, Boerngen-Lacerda R, Andreatini R. Magnesium sulfate and sodium valproate block methylphenidate-induced hyperlocomotion, an animal model of mania. Pharmacol Rep. 2011; 63(1): 64-70.
[11] Safar MM1, Abdallah DM, Arafa NM, Abdel-Aziz MT. Magnesium supplementation enhances the anticonvulsant potential of valproate in pentylenetetrazol-treated rats. Brain Res. 2010 Jun 2; 1334: 58-64. doi: 10.1016/j.brainres.2010.03.076. Epub 2010 Mar 28.

[12] Jin WD, Tian Guosheng, Miao Guodong, Liu Tiebang, Fang Yiru, Jia Fujun, Li Jing, Wang Bo, Lu Luxian, Tan Qingrong, Zhang Ning, Li Lingjiang, Xu Yifeng, Hao Wei. Expert consensus of $m$ agnesium valproate sustained release tablets in treatment for bipolar disorder. J Clin Psychol Med. 2012, 22(5): 289-292.

[13] Li G. Comprison study of magnesium valproate combination with paroxetine in management of treatment-resistant depression(in Chinese). J Clin Psychiatry, 2010, 20(2): 126-127.

[14] Wang XQ, Pei GX, Zhang YL and Zhou HX. Comparative study between magnesium valproate sustained release tablets with sertraline in the treatment of refractory depression(in Chinese). Medical J Chin People Health. 2010, 22(18): 2321-2324.

[15] Cui J. The Effects of magnesium valproate sustained release tablets in treatment-resistant depression. J Practical Med Techniques, 2011, 18(6): 648.

[16] Bian J, Pei SY. The adding effects of magnesium valproate sustained release tablets in treatment-resistant depression(in Chinese). Chin J Health Psychology, 2013, 21(3): 332-333.

[17] Gao XM, Wang QQ, Hou P and Xu CM. Clincal observation of clinical therapy with duloxetine and magnesium valproate sustained release tablets in depression associated with pain(in Chinese). J Neuro Ment Health, 2013, 13(5): 506-507.

[18] Xie ZQ, Deng QX and Zuo JF. Paroxetine combined with magnesium valproate sustained release tablets for refractory depression(in Chinese). J International Med Health Guide, 2013, 19(15): 23772378.

[19] Zhou HX, Wang HY, Ma SJ and Ma XZ. Comparative study of magnesium valproate sustained release tablets with escitalopram in the treatment of refractory depression(in Chinese). J Clin Psychiatry, 2013, 23(4): 220.

[20] Liu YL, Chen YZ, Zhao XZ and Li CS. Comparative study of magnesium valproate sustained release tablets with escitalopram for treatment-resistant depression(in Chinese). J Clin Psychiatry, 2013, 23(4): 288.

[21] Yang SX and Han M. The observation of magnesium valproate sustained release tablets with paroxetine for treatment-resistant depression(in Chinese). J Clin Psychiatry, 2014, 24(4): 282.

[22] Xue XF and Jiang T. Comparative study of escitalopram combing with magnesium valproate sustained release tablets in the treatment of refractory depression(in Chinese). J Chin Rural Health, 2015, 6: 79.

[23] Li XX. The observation of escitalopram combing with magnesium valproate sustained release tablets for treatment-resistant depression(in Chinese). J Chin Prescription Drug, 2014, 14(4): 69-71. 
[24] Lin T. Comparative study of magnesium valproate sustained release tablets with citalopram for treatment-resistant depression(in Chinese). J Clin Psychosomatic Med, 2015, 12(6): 269.

[25] Xie ZQ, Deng QX, Deng HJ and Zou YQ. The Clinical study of venlafaxine combing with magnesium valproate sustained release tablets for treatment-resistant depression(in Chinese). $\mathrm{J}$ Psychologist. 2012, 6: 106-107.

[26] Thase ME. New medications for treatment-resistant depression: a brief review of recent developments. CNS Spectr. 2017 Dec; 22(S1): 39-48. doi: 10.1017/S1092852917000876.

[27] Garay RP, Zarate CA Jr, Charpeaud T, Citrome L, Correll CU, Hameg A, Llorca PM. Investigational drugs in recent clinical trials for treatment-resistant depression. Expert Rev Neurother. 2017 Jun; 17(6): 593-609. doi: 10.1080/14737175.2017.1283217. Epub 2017 Jan 29.

[28] Kagawa S, Mihara K, Suzuki T, Nagai G, Nakamura A, Nemoto K, Kondo T. Both Serum Brain-Derived Neurotrophic
Factor and Interleukin-6 Levels Are Not Associated with Therapeutic Response to Lamotrigine Augmentation Therapy in Treatment-Resistant Depressive Disorder Neuropsychobiology. 2017; 75(3): 145-150. doi: 10.1159/000484665. Epub 2018 Jan 12.

[29] Nugent AC, Carlson PJ, Bain EE, Eckelman W, Herscovitch P , Manji H , Zarate CA , Drevets WC . Mood stabilizer treatment increases serotonin type $1 \mathrm{~A}$ receptor binding in bipolar depression. J Psychopharmacol 2013 Oct; 27 (10): 894-902.

[30] Safar MM, Abdallah DM, Arafa NM, Abdel-Aziz MT. Magnesium supplementation enhances the anticonvulsant potential of valproate in pentylenetetrazol-treated rats. Brain Res. 2010 Jun 2; 1334: 58-64. doi: 10.1016/j. brainres.2010.03.076. Epub 2010 Mar 28.

[31] Gobbi G, Janiri L. Sodium- and magnesium-valproate in vivo modulate glutamatergic and GABAergic synapses in the medial prefrontal cortex. Psychopharmacology (Berl). 2006 Apr; 185(2): 255-62. Epub 2006 Feb 22. 